\title{
Multiple positive solutions for quasilinear elliptic problems with combined critical Sobolev-Hardy terms
}

\section{Yuanyuan $\mathrm{Li}^{1 *}$ (D)}

\section{"Correspondence:}

liyuanyuan@ncwu.edu.cn ${ }^{1}$ School of Mathematics and Statistics, North China University of Water Resources and Electric Power, Zhengzhou, P.R. China

\begin{abstract}
In this paper, we investigate the quasilinear elliptic equations involving multiple critical Sobolev-Hardy terms with Dirichlet boundary conditions on bounded smooth domains $\Omega \subset R^{N}(N \geq 3)$, and prove the multiplicity of positive solutions by employing Ekeland's variational principle and the maximum principle.
\end{abstract}

MSC: 35J20; 35D30

Keywords: Quasilinear elliptic equation; Sobolev-Hardy term; Positive solution; Ekeland's variational principle

\section{Introduction}

In this paper, we consider the following quasilinear elliptic problem:

$$
\begin{cases}-\Delta_{p} u-\mu \frac{|u|^{p-2} u}{|x|^{p}}=\frac{|u|^{p^{*}(a)-2} u}{|x|^{a}}+\frac{|u|^{p^{*}(b)-2} u}{\left|x-x_{0}\right|^{b}}+\lambda \frac{|u|^{q-2} u}{|x|^{s}} & \text { in } \Omega, \\ u=0 & \text { on } \partial \Omega,\end{cases}
$$

where $\Omega \subset R^{N}(N \geq 3)$ is a bounded smooth domain such that the different points $0, x_{0} \in$ $\Omega,-\Delta_{p} u=-\operatorname{div}\left(|\nabla u|^{p-2} \nabla u\right)$ is the $p$-Laplacian of $u ; 0 \leq \mu<\bar{\mu}:=\left(\frac{N-p}{p}\right)^{p}, 1<p<N$ and $\lambda$ is a positive parameter; $0 \leq a \leq b<p, 1 \leq q<p, p^{*}(a)=\frac{p(N-a)}{N-p}, p^{*}(b)=\frac{p(N-b)}{N-p}$. Note that $p^{*}(0)=p^{*}=\frac{N p}{N-p}, p^{*}(p)=p$.

Let $W_{0}^{1, p}(\Omega)$ be the completion of $C_{0}^{\infty}(\Omega)$ with respect to the norm $\left(\int_{\Omega}|\nabla u|^{p} d x\right)^{\frac{1}{p}}$. The energy functional of problem (1) is defined on $W_{0}^{1, p}(\Omega)$ by

$$
\begin{aligned}
J(u)= & \frac{1}{p} \int_{\Omega}\left(|\nabla u|^{p}-\mu \frac{|u|^{p}}{|x|^{p}}\right) d x-\frac{1}{p^{*}(a)} \int_{\Omega} \frac{|u|^{p^{*}(a)}}{|x|^{a}} d x \\
& -\frac{1}{p^{*}(b)} \int_{\Omega} \frac{|u|^{p^{*}(b)}}{\left|x-x_{0}\right|^{b}} d x-\frac{\lambda}{q} \int_{\Omega} \frac{|u|^{q}}{|x|^{s}} d x .
\end{aligned}
$$

Then $J(u) \in C^{1}\left(W_{0}^{1, p}(\Omega), R\right)$. Function $u \in W_{0}^{1, p}(\Omega) \backslash\{0\}$ is said to be a nontrivial solution of (1) if $\left\langle J^{\prime}(u), v\right\rangle=0$ for all $v \in W_{0}^{1, p}(\Omega)$ and a solution of (1) is a critical point of $J(u)$. But the appearance of multiple Sobolev-Hardy terms in (1) makes it difficult to investigate the existence of positive solutions for problem (1).

c) The Author(s) 2019. This article is distributed under the terms of the Creative Commons Attribution 4.0 International License (http://creativecommons.org/licenses/by/4.0/), which permits unrestricted use, distribution, and reproduction in any medium, provided you give appropriate credit to the original author(s) and the source, provide a link to the Creative Commons license, and indicate if changes were made. 
Recall that the functional $J(u)$ satisfies the (PS $)_{c}$ condition if every $(\mathrm{PS})_{c}$ sequence for $J(u)$ has a convergent subsequence, and a sequence $\left\{u_{n}\right\} \subset W_{0}^{1, p}(\Omega)$ is called a (PS) $c$ sequence for $J(u)$ if $J\left(u_{n}\right) \rightarrow c$ and $J^{\prime}\left(u_{n}\right) \rightarrow 0$.

Elliptic equations with critical growth terms have received wide attention in recent years. In a pioneering work, Pohozaev [18] considered the following elliptic problem:

$$
\begin{cases}-\Delta u=|u|^{2^{*}-2} u & \text { in } \Omega, \\ u=0 & \text { on } \partial \Omega\end{cases}
$$

where $\Omega$ is a star-shaped domain with respect to the origin, and obtained that there is no nontrivial solution. However, lower order terms can reverse this situation. Indeed, Brezis and Nirenberg [1] proved the existence of positive solutions for the nonlinear elliptic problem involving the critical Sobolev exponent

$$
\begin{cases}-\Delta u=\lambda u+|u|^{2^{*}-2} u & \text { in } \Omega, \\ u=0 & \text { on } \partial \Omega .\end{cases}
$$

Generalizations of this result can be found in [6], and for multiplicity results for elliptic equations with critical exponents see [7].

As for the elliptic problems involving Hardy terms, Jannelli [13] proved the existence of solutions. This problem was also discussed in $[2,3,8,9]$. The following quasilinear elliptic problems with a singular Hardy term and a critical Sobolev-Hardy term:

$$
\begin{cases}-\Delta_{p} u-\mu \frac{|u|^{p-2} u}{|x|^{p}}=K(x) \frac{|u|^{p^{*}(s)-2} u}{|x|^{s}}+g(x, u) & \text { in } \Omega, \\ u=0 & \text { on } \partial \Omega\end{cases}
$$

have been investigated in recent years, where $K(x)$ is a continuous nonnegative function and $g(x, u)$ is a subcritical perturbation. Kang [14] proved the existence of solutions for problem (2) with $K(x)=1$ and $g(x, u)=\frac{|u|^{q-2} u}{|x|^{t}}$ where $p \leq t<p^{*}(s)$ by using variational methods and the results crucially depend on the parameters $p, q, t, \lambda$, and $\mu$. In [17], Liang consider problem (2) with $K(x)=1$ and derived the existence of infinitely many small solutions by using the concentration compactness principle and a symmetric mountain pass theorem.

Concerning problems with multiple nonlinearities, there has been little research up to now. Here we mention Gao [5] who studied the elliptic problem with combined critical Sobolev-Hardy terms on smooth bounded domain and obtained some existence results by investigating the limit behavior of the PS sequence for the corresponding energy functional. Li [15] has established the complete asymptotic description for any PS sequence $\left\{u_{n}\right\}$ of the associational energy functional and then proved the existence of nontrivial solutions under different assumptions. As for problems involving multiple critical SobolevHardy terms, we refer to articles $[12,16]$.

This paper is devoted to the study of the multiplicity of positive solutions for problem (1) when $a, b, s, \lambda, \mu$ satisfy suitable conditions by using variational methods and some ideas from $[11,12]$. 
Li Boundary Value Problems

(2019) 2019:136

Page 3 of 19

Problem (1) is related to Sobolev-Hardy inequality

$$
\left(\int_{R^{N}} \frac{|u|^{p^{*}(t)}}{|x-a|^{t}} d x\right)^{\frac{p}{p^{*}(t)}} \leq C \int_{R^{N}}|\nabla u|^{p} d x, \quad \forall u \in C_{0}^{\infty}\left(R^{N}\right), a \in R^{N} .
$$

When $t=p, p^{*}(t)=p$, the well-known Hardy inequality holds:

$$
\int_{R^{N}} \frac{|u|^{p}}{|x-a|^{p}} d x \leq \frac{1}{\bar{\mu}} \int_{R^{N}}|\nabla u|^{p} d x, \quad \forall u \in C_{0}^{\infty}\left(R^{N}\right)
$$

where $\bar{\mu}=\left(\frac{N-p}{p}\right)^{p}$ is the best Hardy constant.

In the space $W_{0}^{1, p}(\Omega)$, we employ the following norm if $\mu<\bar{\mu}$ :

$$
\|u\|_{\mu}=\left(\int_{\Omega}\left(|\nabla u|^{p}-\mu \frac{|u|^{p}}{|x|^{p}}\right) d x\right)^{\frac{1}{p}}
$$

Due to Hardy inequality, it is equivalent to the usual norm $\left(\int_{\Omega}|\nabla u|^{p} d x\right)^{\frac{1}{p}}$ of the space $W_{0}^{1, p}(\Omega)$, and

$$
\left(\int_{\Omega}\left(|\nabla u|^{p}-\mu \frac{|u|^{p}}{\left|x-x_{0}\right|^{p}}\right) d x\right)^{\frac{1}{p}}
$$

is also equivalent to the usual norm $\left(\int_{\Omega}|\nabla u|^{p} d x\right)^{\frac{1}{p}}$ of the space $W_{0}^{1, p}(\Omega)$ with $x_{0} \in \Omega$. Hence we can deduce that

$$
\left(\int_{\Omega}\left(|\nabla u|^{p}-\mu \frac{|u|^{p}}{\left|x-x_{0}\right|^{p}}\right) d x\right)^{\frac{1}{p}} \leq \beta\left(\int_{\Omega}\left(|\nabla u|^{p}-\mu \frac{|u|^{p}}{|x|^{p}}\right) d x\right)^{\frac{1}{p}}
$$

where $\beta$ is a constant.

Set

$$
A_{\mu, t}(\Omega)=\inf _{u \in W_{0}^{1, p}(\Omega) \backslash\{0\}} \frac{\int_{\Omega}\left(|\nabla u|^{p}-\mu \frac{|u|^{p}}{|x-a|^{p}}\right) d x}{\left(\int_{\Omega} \frac{|u|^{p^{*}(t)}}{|x-a|^{t}} d x\right)^{\frac{p}{p^{*}(t)}}}, \quad a \in \Omega .
$$

Whenever $A_{\mu, t}$ is independent of $\Omega \subset R^{N}$, we will simple denote $A_{\mu, t}(\Omega)=A_{\mu, t}\left(R^{N}\right)=A_{\mu, t}$. Therefore we conclude that

$$
\int_{\Omega} \frac{|u|^{p^{*}(b)}}{\left|x-x_{0}\right|^{b}} d x \leq \frac{\left(\int_{\Omega}\left(|\nabla u|^{p}-\mu \frac{|u|^{p}}{\left|x-x_{0}\right|^{p}}\right) d x\right)^{\frac{p^{*}(b)}{p}}}{A_{\mu, b}^{\frac{p^{*}(b)}{p}}} \leq \frac{\beta^{p^{*}(b)}\|u\|_{\mu}^{p^{*}(b)}}{A_{\mu, b}^{\frac{p^{*}(b)}{p}}} .
$$

Let

$$
\begin{aligned}
\Lambda_{0}= & \min \left\{\left(\frac{p-q}{2 \beta^{*}(b)\left(p^{*}(b)-q\right)}\right)^{\frac{p-q}{p^{*}(b)-p}} \frac{p-p^{*}(b)}{q-p^{*}(b)}\left(\frac{N \omega_{N} R_{0}^{N-s}}{N-s}\right)^{\frac{p^{*}(s)-q}{-p^{*}(s)}} A_{\mu, s}^{\frac{q}{p}} A_{\mu, b}^{\frac{(N-b)(p-q)}{p(p-b)}},\right. \\
& \left.\left(\frac{p-q}{2\left(p^{*}(a)-q\right)}\right)^{\frac{p-q}{p^{*}(a)-p}} \frac{p-p^{*}(b)}{q-p^{*}(b)}\left(\frac{N \omega_{N} R_{0}^{N-s}}{N-s}\right)^{\frac{p^{*}(s)-q}{-p^{*}(s)}} A_{\mu, s}^{\frac{q}{p}} A_{\mu, a}^{\frac{(N-a)(p-q)}{p(p-a)}}\right\}
\end{aligned}
$$


and

$$
\Lambda_{1}=\min \left\{\frac{p-a}{p(N-a)} A_{\mu, a}^{\frac{N-a}{p-a}}, \frac{p-b}{p(N-b)} A_{0, b}^{\frac{N-b}{p-b}}\right\} .
$$

Now we give our main result:

Theorem 1.1 If $N \geq 3,0 \leq \mu<\bar{\mu}, 0 \leq a \leq b<p, 0 \leq s<p, 1 \leq q<p$, then we have the following results:

(i) If $\lambda \in\left(0, \Lambda_{0}\right)$, then (1) has at least one positive solution in $W_{0}^{1, p}(\Omega)$.

(ii) If $\lambda \in\left(0, \frac{q}{p} \Lambda_{0}\right)$, then (1) has at least two positive solutions in $W_{0}^{1, p}(\Omega)$.

This paper is organized as follows. In Sect. 2, we narrate some useful preliminary knowledge and some properties of Nehari manifolds. In Sect. 3, the multiplicity of positive weak solutions is verified.

Throughout this paper, various positive constants will be denoted by $c$, and $d x$ in integrals will be omitted for convenience.

\section{Preliminary knowledge and main results}

Since the functional $J(u)$ is not bounded from below on $W_{0}^{1, p}(\Omega)$, we will work on a Nehari manifold. For $\lambda>0$, we define

$$
N_{\lambda}=\left\{u \in W_{0}^{1, p}(\Omega) \backslash\{0\}:\left\langle J^{\prime}(u), u\right\rangle=0\right\} .
$$

We recall that any nonzero solution of (1) belongs to $N_{\lambda}$. Moreover, by definition, we have that $u \in N_{\lambda}$ if and only if

$$
\|u\|_{\mu} \neq 0 \text { and }\|u\|_{\mu}^{p}-\int_{\Omega} \frac{|u|^{p^{*}(a)}}{|x|^{a}}-\int_{\Omega} \frac{|u|^{p^{*}(b)}}{\left|x-x_{0}\right|^{b}}-\lambda \int_{\Omega} \frac{|u|^{q}}{|x|^{s}}=0 .
$$

Lemma 2.1 The functional $J(u)$ is coercive and bounded from below on $N_{\lambda}$.

Proof For $u \in N_{\lambda}$, we have

$$
\begin{aligned}
J(u) & =\frac{1}{p}\|u\|_{\mu}^{p}-\frac{1}{p^{*}(a)} \int_{\Omega} \frac{|u|^{p^{*}(a)}}{|x|^{a}}-\frac{1}{p^{*}(b)} \int_{\Omega} \frac{|u|^{p^{*}(b)}}{\left|x-x_{0}\right|^{b}}-\frac{\lambda}{q} \int_{\Omega} \frac{|u|^{q}}{|x|^{s}} \\
& \geq \frac{1}{p}\|u\|_{\mu}^{p}-\frac{\lambda}{q} \int_{\Omega} \frac{|u|^{q}}{|x|^{s}}-\frac{1}{p^{*}(b)}\left(\int_{\Omega} \frac{|u|^{p^{*}(a)}}{|x|^{a}}+\int_{\Omega} \frac{|u|^{p^{*}(b)}}{\left|x-x_{0}\right|^{b}}\right) \\
& =\left(\frac{1}{p}-\frac{1}{p^{*}(b)}\right)\|u\|_{\mu}^{p}-\lambda\left(\frac{1}{q}-\frac{1}{p^{*}(b)}\right) \int_{\Omega} \frac{|u|^{q}}{|x|^{s}} \\
& \geq\left(\frac{1}{p}-\frac{1}{p^{*}(b)}\right)\|u\|_{\mu}^{p}-\lambda\left(\frac{1}{q}-\frac{1}{p^{*}(b)}\right)\left(\int_{\Omega} \frac{|u|^{p^{*}(s)}}{|x|^{s}}\right)^{\frac{q}{p^{*(s)}}}\left(\int_{\Omega}|x|^{-s}\right)^{\frac{p^{*}(s)-q}{p^{*(s)}}} .
\end{aligned}
$$

Set $R_{0}$ be a positive constant such that $\Omega \subset B\left(0 ; R_{0}\right)$, where $B\left(0 ; R_{0}\right)=\left\{x \in R^{N}:|x|<R_{0}\right\}$. Since

$$
\left(\int_{\Omega}|x|^{-s}\right)^{\frac{p^{*}(s)-q}{p^{*}(s)}} \leq\left(N \omega_{N} \int_{0}^{R_{0}} r^{-s+N-1} d r\right)^{\frac{p^{*}(s)-q}{p^{*}(s)}}=\left(\frac{N \omega_{N} R_{0}^{N-s}}{N-s}\right)^{\frac{p^{*}(s)-q}{p^{*}(s)}}
$$


where $\omega_{N}=\frac{2 \pi^{\frac{N}{2}}}{N \Gamma\left(\frac{N}{2}\right)}$ is the volume of the unit ball in $R^{N}$, we have

$$
\left(\int_{\Omega} \frac{|u|^{p^{*}(s)}}{|x|^{s}}\right)^{\frac{q}{p^{*}(s)}} \leq A_{\mu, s}^{-\frac{q}{p}}\|u\|_{\mu}^{q}
$$

Thus combining with (4), we get that

$$
J(u) \geq \frac{p-b}{p(N-b)}\|u\|_{\mu}^{p}-\lambda \frac{p^{*}(b)-q}{q p^{*}(b)}\left(\frac{N \omega_{N} R_{0}^{N-s}}{N-s}\right)^{\frac{p^{*}(s)-q}{p^{*}(s)}} A_{\mu, s}^{-\frac{q}{p}}\|u\|_{\mu}^{q} .
$$

Since $0 \leq b, s<p$ and $1 \leq q<p$, $J(u)$ is coercive and bounded below on $N_{\lambda}$.

Define $\phi_{\lambda}: W_{0}^{1, p}(\Omega) \rightarrow R$ by $\phi_{\lambda}(u)=\left\langle J^{\prime}(u), u\right\rangle$, that is,

$$
\phi_{\lambda}(u)=\|u\|_{\mu}^{p}-\int_{\Omega} \frac{|u|^{p^{*}(a)}}{|x|^{a}}-\int_{\Omega} \frac{|u|^{p^{*}(b)}}{\left|x-x_{0}\right|^{b}}-\lambda \int_{\Omega} \frac{|u|^{q}}{|x|^{s}} .
$$

Note that $\phi_{\lambda}$ is of class $C^{1}$ with

$$
\left\langle\phi_{\lambda}^{\prime}(u), u\right\rangle=p\|u\|_{\mu}^{p}-p^{*}(a) \int_{\Omega} \frac{|u|^{p^{*}(a)}}{|x|^{a}}-p^{*}(b) \int_{\Omega} \frac{|u|^{p^{*}(b)}}{\left|x-x_{0}\right|^{b}}-\lambda q \int_{\Omega} \frac{|u|^{q}}{|x|^{s}} .
$$

Furthermore, if $u \in N_{\lambda}$, then from (3) and (6), we have

$$
\begin{aligned}
\left\langle\phi_{\lambda}^{\prime}(u), u\right\rangle= & p\|u\|_{\mu}^{p}-p^{*}(a) \int_{\Omega} \frac{|u|^{p^{*}(a)}}{|x|^{a}}-p^{*}(b) \int_{\Omega} \frac{|u|^{p^{*}(b)}}{\left|x-x_{0}\right|^{b}} \\
& -q\left(\|u\|_{\mu}^{p}-\int_{\Omega} \frac{|u|^{p^{*}(a)}}{|x|^{a}}-\int_{\Omega} \frac{|u|^{p^{*}(b)}}{\left|x-x_{0}\right|^{b}}\right) \\
= & (p-q)\|u\|_{\mu}^{p}-\left(p^{*}(a)-q\right) \int_{\Omega} \frac{|u|^{p^{*}(a)}}{|x|^{a}} \\
& -\left(p^{*}(b)-q\right) \int_{\Omega} \frac{|u|^{p^{*}(b)}}{\left|x-x_{0}\right|^{b}}
\end{aligned}
$$

and

$$
\begin{aligned}
\left\langle\phi_{\lambda}^{\prime}(u), u\right\rangle= & p\|u\|_{\mu}^{p}-p^{*}(a) \int_{\Omega} \frac{|u|^{*^{*}(a)}}{|x|^{a}}-\lambda q \int_{\Omega} \frac{|u|^{q}}{|x|^{s}} \\
& -p^{*}(b)\left(\|u\|_{\mu}^{p}-\int_{\Omega} \frac{|u|^{p^{*}(a)}}{|x|^{a}}-\lambda \int_{\Omega} \frac{|u|^{q}}{|x|^{s}}\right) \\
= & \left(p-p^{*}(b)\right)\|u\|_{\mu}^{p}-\left(p^{*}(a)-p^{*}(b)\right) \int_{\Omega} \frac{|u|^{p^{*}(a)}}{|x|^{a}} \\
& -\lambda\left(q-p^{*}(b)\right) \int_{\Omega} \frac{|u|^{q}}{|x|^{s}} .
\end{aligned}
$$


Now we split $N_{\lambda}$ into three sets:

$$
\begin{aligned}
& N_{\lambda}^{+}=\left\{u \in N_{\lambda}:\left\langle\phi_{\lambda}^{\prime}(u), u\right\rangle>0\right\}, \\
& N_{\lambda}^{0}=\left\{u \in N_{\lambda}:\left\langle\phi_{\lambda}^{\prime}(u), u\right\rangle=0\right\}, \\
& N_{\lambda}^{-}=\left\{u \in N_{\lambda}:\left\langle\phi_{\lambda}^{\prime}(u), u\right\rangle<0\right\} .
\end{aligned}
$$

The following result shows that minimizers on $N_{\lambda}$ are the usual critical points for $J(u)$.

Lemma 2.2 Suppose that $u_{0}$ is a local minimizer of $J(u)$ on $N_{\lambda}$ and $u_{0} \notin N_{\lambda}^{0}$, then $J^{\prime}\left(u_{0}\right)=0$ in $\left(W_{0}^{1, p}(\Omega)\right)^{-1}$.

Proof It is easy to see that there exists a neighborhood $U$ of $u_{0}$ in $W_{0}^{1, p}(\Omega)$ such that

$$
J\left(u_{0}\right)=\min _{u \in U \cap N_{\lambda}} J(u)=\min _{u \in U \backslash\{0\}, \phi_{\lambda}(u)=0} J(u) .
$$

Furthermore, by the Lagrange Multipliers Theorem, there exists $\rho \in R$ such that $J^{\prime}\left(u_{0}\right)=$ $\rho \phi_{\lambda}\left(u_{0}\right)$. Then, since $u_{0} \in N_{\lambda}$, we get

$$
0=\left\langle J^{\prime}\left(u_{0}\right), u_{0}\right\rangle=\rho\left\langle\phi_{\lambda}^{\prime}\left(u_{0}\right), u_{0}\right\rangle
$$

Now $u_{0} \notin N_{\lambda}^{0}$, thus $\rho=0$, and consequently $J^{\prime}\left(u_{0}\right)=0$ in $\left(W_{0}^{1, p}(\Omega)\right)^{-1}$.

Motivated by the above result, we will get conditions for $N_{\lambda}^{0}=\emptyset$.

Lemma 2.3 If $\lambda \in\left(0, \Lambda_{0}\right)$, then $N_{\lambda}^{0}=\emptyset$, where $\Lambda_{0}$ is given in the introduction .

Proof We argue by contradiction. Suppose that there exists a $\lambda \in\left(0, \Lambda_{0}\right)$ such that $N_{\lambda}^{0} \neq \emptyset$, then from (9),

$$
\begin{aligned}
0 & \leq\|u\|_{\mu}^{p} \\
& =\frac{p^{*}(a)-p^{*}(b)}{p-p^{*}(b)} \int_{\Omega} \frac{|u|^{p^{*}(a)}}{|x|^{a}}+\lambda \frac{q-p^{*}(b)}{p-p^{*}(b)} \int_{\Omega} \frac{|u|^{q}}{|x|^{s}} \\
& \leq \lambda \frac{q-p^{*}(b)}{p-p^{*}(b)} \int_{\Omega} \frac{|u|^{q}}{|x|^{s}} \\
& \leq \lambda \frac{q-p^{*}(b)}{p-p^{*}(b)}\left(\frac{N \omega_{N} R_{0}^{N-s}}{N-s}\right)^{\frac{p^{*}(s)-q}{p^{*}(s)}} A_{\mu, s}^{-\frac{q}{p}}\|u\|_{\mu}^{q},
\end{aligned}
$$

which implies

$$
\|u\|_{\mu} \leq\left(\lambda \frac{q-p^{*}(b)}{p-p^{*}(b)}\left(\frac{N \omega_{N} R_{0}^{N-s}}{N-s}\right)^{\frac{p^{*}(s)-q}{p^{*}(s)}} A_{\mu, s}^{-\frac{q}{p}}\right)^{\frac{1}{p-q}} .
$$

Again by using (7), Hölder and Sobolev-Hardy inequalities, we have

$$
\begin{aligned}
0 & \leq\|u\|_{\mu}^{p} \\
& =\frac{p^{*}(a)-q}{p-q} \int_{\Omega} \frac{|u|^{p^{*}(a)}}{|x|^{a}}+\frac{p^{*}(b)-q}{p-q} \int_{\Omega} \frac{|u|^{p^{*}(b)}}{\left|x-x_{0}\right|^{b}}
\end{aligned}
$$




$$
\begin{aligned}
& \leq \frac{p^{*}(a)-q}{p-q} \frac{\|u\|_{\mu}^{p^{*}(a)}}{A_{\mu, a}^{\frac{p^{*}(a)}{p}}}+\frac{p^{*}(b)-q}{p-q} \frac{\beta^{p^{*}(b)}\|u\|_{\mu}^{p^{*}(b)}}{A_{\mu, b}^{\frac{p^{*}(b)}{p}}} \\
& \leq 2 \max \left\{\frac{p^{*}(a)-q}{p-q} \frac{\|u\|_{\mu}^{p^{*}(a)}}{A_{\mu, a}^{\frac{p^{*}(a)}{p}}}, \frac{p^{*}(b)-q}{p-q} \frac{\beta^{p^{*}(b)}\|u\|_{\mu}^{p^{*}(b)}}{A_{\mu, b}^{\frac{p^{*}(b)}{p}}}\right\} .
\end{aligned}
$$

Now we distinguish two cases:

$$
\text { Case 1. } \frac{p^{*}(a)-q}{p-q} \frac{\|u\|_{\mu}^{p^{*}(a)}}{A_{\mu, a}^{\frac{p^{*}(a)}{p}}} \leq \frac{p^{*}(b)-q}{p-q} \frac{\beta^{p^{*}(b)} \| u u_{\mu}^{p^{*}(b)}}{A_{\mu, b}^{\frac{p^{*}(b)}{p}}} .
$$

It is easy to calculate that

$$
\|u\|_{\mu} \geq\left(\frac{p-q}{2 \beta^{p^{*}(b)}\left(p^{*}(b)-q\right)} A_{\mu, b}^{\frac{p^{*}(b)}{p}}\right)^{\frac{1}{p^{*}(b)-p}} .
$$

Combining with (11), we conclude that

$$
\lambda \geq\left(\frac{p-q}{2 \beta^{p^{*}(b)}\left(p^{*}(b)-q\right)}\right)^{\frac{p-q}{p^{*}(b)-p}} \frac{p-p^{*}(b)}{q-p^{*}(b)}\left(\frac{N \omega_{N} R_{0}^{N-s}}{N-s}\right)^{\frac{p^{*}(s)-q}{-p^{*}(s)}} A_{\mu, s}^{\frac{q}{p}} A_{\mu, b}^{\frac{(N-b)(p-q)}{p(p-b)}} .
$$

Case 2. $\frac{p^{*}(a)-q}{p-q} \frac{\|u\|_{p}^{p^{*}(a)}}{A_{\mu, a}^{\frac{p^{*}(a)}{p}}}>\frac{p^{*}(b)-q}{p-q} \frac{\beta^{p^{*}(b)} \| u u_{\mu}^{p^{*}(b)}}{A_{\mu, b}^{\frac{p^{*}(b)}{p}}}$.

As in Case 1, one obtains that

$$
\lambda>\left(\frac{p-q}{2\left(p^{*}(a)-q\right)}\right)^{\frac{p-q}{p^{*}(a)-p}} \frac{p-p^{*}(b)}{q-p^{*}(b)}\left(\frac{N \omega_{N} R_{0}^{N-s}}{N-s}\right)^{\frac{p^{*}(s)-q}{-p^{*}(s)}} A_{\mu, s}^{\frac{q}{p}} A_{\mu, a}^{\frac{(N-a)(p-q)}{p(p-a)}} .
$$

Hence $\lambda \geq \Lambda_{0}$, which contradicts $\lambda \in\left(0, \Lambda_{0}\right)$. Thus $N_{\lambda}^{0}=\emptyset$.

Lemma 2.4 If $\lambda \in\left(0, \Lambda_{0}\right)$, then for each $u \in W_{0}^{1, p}(\Omega) \backslash\{0\}$, the set $\{\tau u: \tau>0\}$ intersects $N_{\lambda}$ exactly twice. More specifically, there exist a unique $\tau^{-}=\tau^{-}(u)>0$ such that $\tau^{-} u \in N_{\lambda}^{-}$and a unique $\tau^{+}=\tau^{+}(u)>0$ such that $\tau^{+} u \in N_{\lambda}^{+}$. Moreover, $\tau^{+}<\tau_{\max }<\tau^{-}$and

$$
J\left(\tau^{+} u\right)=\inf _{0 \leq \tau \leq \tau_{\max }} J(\tau u), \quad J\left(\tau^{-} u\right)=\sup _{\tau \geq \tau_{\max }} J(\tau u) .
$$

Proof The proof is similar to that of Lemma 2.7 in [11], and we omit it here.

From Lemma 2.3 we obtain that $N_{\lambda}=N_{\lambda}^{+} \cup N_{\lambda}^{-}$for all $\lambda \in\left(0, \Lambda_{0}\right)$. Furthermore, by Lemma 2.4 it follows that $N_{\lambda}^{+}$and $N_{\lambda}^{-}$are nonempty and, by Lemma 2.1, we may define

$$
\alpha_{\lambda}=\inf _{u \in N_{\lambda}} J(u), \quad \alpha_{\lambda}^{+}=\inf _{u \in N_{\lambda}^{+}} J(u), \quad \alpha_{\lambda}^{-}=\inf _{u \in N_{\lambda}^{-}} J(u) .
$$

\section{Lemma 2.5}

(i) If $\lambda \in\left(0, \Lambda_{0}\right)$, then we have $\alpha_{\lambda} \leq \alpha_{\lambda}^{+}<0$.

(ii) If $\lambda \in\left(0, \frac{q}{p} \Lambda_{0}\right)$, then there exists some positive constant $d_{0}$ such that $\alpha_{\lambda}^{-}>d_{0}$.

In particular, for each $\lambda \in\left(0, \frac{q}{p} \Lambda_{0}\right)$, we have that $\alpha_{\lambda}^{+}<0<\alpha_{\lambda}^{-}$. 
Li Boundary Value Problems

(2019) 2019:136

Page 8 of 19

Proof (i) It is enough to prove that there exists $c>0$ such that $\alpha_{\lambda}^{+}<-c<0$. Let $u \in N_{\lambda}^{+}$. Then from (7), we have

$$
\|u\|_{\mu}^{p}>\frac{p^{*}(a)-q}{p-q} \int_{\Omega} \frac{|u|^{p^{*}(a)}}{|x|^{a}}+\frac{p^{*}(b)-q}{p-q} \int_{\Omega} \frac{|u|^{p^{*}(b)}}{\left|x-x_{0}\right|^{b}} .
$$

Therefore for $u \in N_{\lambda}^{+}$, we get

$$
\begin{aligned}
J(u)= & \left(\frac{1}{p}-\frac{1}{q}\right)\|u\|_{\mu}^{p}-\left(\frac{1}{p^{*}(a)}-\frac{1}{q}\right) \int_{\Omega} \frac{|u|^{p^{*}(a)}}{|x|^{a}}-\left(\frac{1}{p^{*}(b)}-\frac{1}{q}\right) \int_{\Omega} \frac{|u|^{p^{*}(b)}}{\left|x-x_{0}\right|^{b}} \\
< & \left\{\left(\frac{1}{p}-\frac{1}{q}\right) \frac{p^{*}(a)-q}{p-q}-\left(\frac{1}{p^{*}(a)}-\frac{1}{q}\right)\right\} \int_{\Omega} \frac{|u|^{p^{*}(a)}}{|x|^{a}} \\
& +\left\{\left(\frac{1}{p}-\frac{1}{q}\right) \frac{p^{*}(b)-q}{p-q}-\left(\frac{1}{p^{*}(b)}-\frac{1}{q}\right)\right\} \int_{\Omega} \frac{|u|^{p^{*}(b)}}{\left|x-x_{0}\right|^{b}} \\
= & \frac{\left(p^{*}(a)-q\right)\left(p-p^{*}(a)\right)}{p q p^{*}(a)} \int_{\Omega} \frac{|u|^{p^{*}(a)}}{|x|^{a}}+\frac{\left(p^{*}(b)-q\right)\left(p-p^{*}(b)\right)}{p q p^{*}(b)} \int_{\Omega} \frac{|u|^{p^{*}(b)}}{\left|x-x_{0}\right|^{b}} \\
< & 0,
\end{aligned}
$$

where $q<p<p^{*}(b) \leq p^{*}(a)$. Therefore, from the definition of $\alpha_{\lambda}$ and $\alpha_{\lambda}^{+}$, we can deduce that $\alpha_{\lambda} \leq \alpha_{\lambda}^{+}<0$.

(ii) Let $u \in N_{\lambda}^{-}$. By (7),

$$
\|u\|_{\mu}^{p}<\frac{p^{*}(a)-q}{p-q} \int_{\Omega} \frac{|u|^{p^{*}(a)}}{|x|^{a}}+\frac{p^{*}(b)-q}{p-q} \int_{\Omega} \frac{|u|^{p^{*}(b)}}{\left|x-x_{0}\right|^{b}} .
$$

Thus from the Sobolev-Hardy inequality, we get

$$
\begin{aligned}
\|u\|_{\mu}^{p} & <\frac{p^{*}(a)-q}{p-q} A_{\mu, a}^{-\frac{p^{*}(a)}{p}}\|u\|_{\mu}^{p^{*}(a)}+\frac{p^{*}(b)-q}{p-q} A_{\mu, b}^{-\frac{p^{*}(b)}{p}} \beta^{p^{*}(b)}\|u\|_{\mu}^{p^{*}(b)} \\
& \leq 2 \max \left\{\frac{p^{*}(a)-q}{p-q} A_{\mu, a}^{-\frac{p^{*}(a)}{p}}\|u\|_{\mu}^{p^{*}(a)}, \frac{p^{*}(b)-q}{p-q} A_{\mu, b}^{-\frac{p^{*}(b)}{p}} \beta^{p^{*}(b)}\|u\|_{\mu}^{p^{*}(b)}\right\} .
\end{aligned}
$$

Case 1. $\frac{p^{*}(a)-q}{p-q} A_{\mu, a}^{-\frac{p^{*}(a)}{p}}\|u\|_{\mu}^{p^{*}(a)} \leq \frac{p^{*}(b)-q}{p-q} A_{\mu, b}^{-\frac{p^{*}(b)}{p}} \beta^{p^{*}(b)}\|u\|_{\mu}^{p^{*}(b)}$.

It is easy to calculate that for all $u \in N_{\lambda}^{-}$,

$$
\|u\|_{\mu} \geq\left(\frac{p-q}{2 \beta^{p^{*}(b)}\left(p^{*}(b)-q\right)} A_{\mu, b}^{\frac{p^{*}(b)}{p}}\right)^{\frac{1}{p^{*}(b)-p}} .
$$

From (5) and (12), we obtain

$$
\begin{aligned}
& J(u) \geq \frac{p-b}{p(N-b)}\|u\|_{\mu}^{p}-\lambda \frac{p^{*}(b)-q}{q p^{*}(b)}\left(\frac{N \omega_{N} R_{0}^{N-s}}{N-s}\right)^{\frac{p^{*}(s)-q}{p^{*}(s)}} A_{\mu, s}^{-\frac{q}{p}}\|u\|_{\mu}^{q} \\
& \geq\left(\frac{p-q}{2 \beta^{p^{*}(b)}\left(p^{*}(b)-q\right)} A_{\mu, b}^{\frac{p^{*}(b)}{p}}\right)^{\frac{q}{p^{*}(b)-p}} \\
& \times\left\{\frac{p-b}{p(N-b)}\left(\frac{p-q}{2 \beta^{*}(b)\left(p^{*}(b)-q\right)} A_{\mu, b}^{\frac{p^{*}(b)}{p}}\right)^{\frac{p-q}{p^{*}(b)-p}}\right.
\end{aligned}
$$




$$
\begin{aligned}
& \left.-\lambda \frac{p^{*}(b)-q}{q p^{*}(b)}\left(\frac{N \omega_{N} R_{0}^{N-s}}{N-s}\right)^{\frac{p^{*}(s)-q}{p^{*}(s)}} A_{\mu, s}^{-\frac{q}{p}}\right\}
\end{aligned}
$$

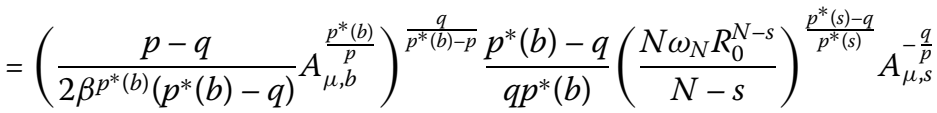

$$
\begin{aligned}
& \times\left\{\frac{p-b}{p(N-b)}\left(\frac{p-q}{2 \beta^{*}(b)\left(p^{*}(b)-q\right)} A_{\mu, b}^{\frac{p^{*}(b)}{p}}\right)^{\frac{p-q}{p^{*}(b)-p}} \frac{q p^{*}(b)}{p^{*}(b)-q}\right. \\
& \left.\times\left(\frac{N \omega_{N} R_{0}^{N-s}}{N-s}\right)^{-\frac{p^{*}(s)-q}{p^{*}(s)}} A_{\mu, s}^{\frac{q}{p}}-\lambda\right\} \\
& \geq\left(\frac{q}{p} \Lambda_{0}-\lambda\right)\left(\frac{p-q}{2 \beta^{p^{*}(b)}\left(p^{*}(b)-q\right)} A_{\mu, b}^{\frac{p^{*}(b)}{p}}\right)^{\frac{q}{p^{*}(b)-p}} \frac{p^{*}(b)-q}{q p^{*}(b)}\left(\frac{N \omega_{N} R_{0}^{N-s}}{N-s}\right)^{\frac{p^{*}(s)-q}{p^{*}(s)}} A_{\mu, s}^{-\frac{q}{p}} \\
& >0 \text {. }
\end{aligned}
$$

Case 2. $\frac{p^{*}(a)-q}{p-q} A_{\mu, a}^{-\frac{p^{*}(a)}{p}}\|u\|_{\mu}^{p^{*}(a)}>\frac{p^{*}(b)-q}{p-q} A_{\mu, b}^{-\frac{p^{*}(b)}{p}}\|u\|_{\mu}^{p^{*}(b)}$.

It is easy to calculate that for all $u \in N_{\lambda}^{-}$

$$
\|u\|_{\mu} \geq\left(\frac{p-q}{2\left(p^{*}(a)-q\right)} A_{\mu, a}^{\frac{p^{*}(a)}{p}}\right)^{\frac{1}{p^{*}(a)-p}} .
$$

With (5), we deduce that

$$
\begin{aligned}
J(u) \geq & \left(\frac{p-q}{2\left(p^{*}(a)-q\right)} A_{\mu, a}^{\frac{p^{*}(a)}{p}}\right)^{\frac{q}{p^{*}(a)-p}} \\
& \times\left\{\frac{p-b}{p(N-b)}\left(\frac{p-q}{2\left(p^{*}(a)-q\right)} A_{\mu, a}^{\frac{p^{*}(a)}{p}}\right)^{\frac{p-q}{p^{*}(a)-p}}\right. \\
& \left.-\lambda \frac{p^{*}(b)-q}{q p^{*}(b)}\left(\frac{N \omega_{N} R_{0}^{N-s}}{N-s}\right)^{\frac{p^{*}(s)-q}{p^{*}(s)}} A_{\mu, s}^{-\frac{q}{p}}\right\} \\
= & \left(\frac{p-q}{2\left(p^{*}(b)-q\right)} A_{\mu, a}^{\frac{p^{*}(a)}{p}}\right)^{\frac{q}{p^{*}(a)-p}} \frac{p^{*}(b)-q}{q p^{*}(b)}\left(\frac{N \omega_{N} R_{0}^{N-s}}{N-s}\right)^{\frac{p^{*}(s)-q}{p^{*}(s)}} A_{\mu, s}^{-\frac{q}{p}} \\
& \times\left\{\frac{p-b}{p(N-b)}\left(\frac{p-q}{2\left(p^{*}(a)-q\right)} A_{\mu, a}^{\frac{p^{*}(a)}{p}}\right)^{\frac{p-q}{p^{*}(a)-p}}\right. \\
& \left.\times \frac{q p^{*}(b)}{p^{*}(b)-q}\left(\frac{N \omega_{N} R_{0}^{N-s}}{N-s}\right)^{-\frac{p^{*}(s)-q}{p^{*}(s)}} A_{\mu, s}^{\frac{q}{p}}-\lambda\right\} \\
\geq & \left(\frac{q}{p} \Lambda_{0}-\lambda\right)\left(\frac{p-q}{2\left(p^{*}(b)-q\right)} A_{\mu, a}^{\frac{p^{*}(a)}{p}}\right)^{\frac{q}{p^{*}(a)-p}} \frac{p^{*}(b)-q}{q p^{*}(b)}\left(\frac{N \omega_{N} R_{0}^{N-s}}{N-s}\right)^{\frac{p^{*}(s)-q}{p^{*}(s)}} A_{\mu, s}^{-\frac{q}{p}}
\end{aligned}
$$$$
>0 \text {. }
$$

So if $\lambda \in\left(0, \frac{q}{p} \Lambda_{0}\right)$, then $J(u)>d_{0}$ for all $u \in N_{\lambda}^{-}$for some positive constant $d_{0}$. 
Remark 1 If $\lambda \in\left(0, \frac{q}{p} \Lambda_{0}\right)$, then by Lemmas 2.4 and 2.5 , for each $u \in W_{0}^{1, p}(\Omega) \backslash\{0\}$, we can easily deduce that

$$
\tau^{-} u \in N_{\lambda}^{-} \quad \text { and } \quad J\left(\tau^{-} u\right)=\sup _{\tau \geq 0} J(\tau u) \geq \alpha_{\lambda}^{-}>0 .
$$

\section{Proof of the main result}

\section{Lemma 3.1}

(i) If $\lambda \in\left(0, \Lambda_{0}\right)$, then $J(u)$ has a $(P S)_{\alpha_{\lambda}}$ sequence $\left\{u_{n}\right\} \subset N_{\lambda}$.

(ii) If $\lambda \in\left(0, \frac{q}{p} \Lambda_{0}\right)$, then $J(u)$ has a $(\mathrm{PS})_{\alpha_{\lambda}^{-}}$sequence $\left\{u_{n}\right\} \subset N_{\lambda}^{-}$.

Proof The proof is similar to that of Proposition 3.3 in [11], and we omit it here.

Now we use the Ekeland's variational principle [4] to get the following results.

Theorem 3.2 If $\lambda \in\left(0, \Lambda_{0}\right)$, then there exists $u_{\lambda} \in N_{\lambda}^{+}$such that

(i) $J\left(u_{\lambda}\right)=\alpha_{\lambda}=\alpha_{\lambda}^{+}$;

(ii) $u_{\lambda}$ is a positive solution for problem (1);

(iii) $\left\|u_{\lambda}\right\|_{\mu} \rightarrow 0$ as $\lambda \rightarrow 0^{+}$.

Proof By Lemma 3.1(i), there exists a minimizing sequence $\left\{u_{n}\right\} \subset N_{\lambda}$ such that

$$
J\left(u_{n}\right)=\alpha_{\lambda}+o(1) \quad \text { and } \quad J^{\prime}\left(u_{n}\right)=o(1) \quad \text { in }\left(W_{0}^{1, p}(\Omega)\right)^{-1} .
$$

Since $J(u)$ is coercive on $N_{\lambda}$, we obtain that $\left\{u_{n}\right\}$ is bounded in $W_{0}^{1, p}(\Omega)$. Thus, passing to a subsequence if necessary, there exists $u_{\lambda} \in W_{0}^{1, p}(\Omega)$ such that as $n \rightarrow \infty$,

$$
\begin{cases}u_{n} \rightarrow u_{\lambda} & \text { weakly in } W_{0}^{1, p}(\Omega), \\ u_{n} \rightarrow u_{\lambda} & \text { weakly in } L^{p^{*}(t)}\left(\Omega,|x|^{-t}\right) \text { for } 0 \leq t<p, \\ u_{n} \rightarrow u_{\lambda} & \text { strongly in } L^{q}\left(\Omega,|x|^{-s}\right) \text { for } 1 \leq q<p^{*}(s), \\ u_{n} \rightarrow u_{\lambda} & \text { a.e. in } \Omega .\end{cases}
$$

From (13) and (14), it is easy to see that $u_{\lambda}$ is a solution of (1). Furthermore, from $u_{n} \in N_{\lambda}$ and (4), we deduce that

$$
\begin{aligned}
\lambda \int_{\Omega} \frac{\left|u_{n}\right|^{q}}{|x|^{s}} & \geq\left(\frac{1}{p}-\frac{1}{p^{*}(b)}\right) \frac{q p^{*}(b)}{p^{*}(b)-q}\left\|u_{n}\right\|_{\mu}^{p}-\frac{q p^{*}(b)}{p^{*}(b)-q} J\left(u_{n}\right) \\
& =\frac{q\left(p^{*}(b)-p\right)}{p\left(p^{*}(b)-q\right)}\left\|u_{n}\right\|_{\mu}^{p}-\frac{q p^{*}(b)}{p^{*}(b)-q} J\left(u_{n}\right) \\
& \geq-\frac{q p^{*}(b)}{p^{*}(b)-q} J\left(u_{n}\right) .
\end{aligned}
$$

Let $n \rightarrow \infty$ in (15). Then from (13)-(14) and since $\alpha_{\lambda}<0$ by Lemma 2.5(i), we get

$$
\lambda \int_{\Omega} \frac{\left|u_{\lambda}\right|^{q}}{|x|^{s}} \geq-\frac{q p^{*}(b)}{p^{*}(b)-q} \alpha_{\lambda}>0
$$

Thus $u_{\lambda} \neq 0$. Since $J^{\prime}\left(u_{\lambda}\right)=0$, it follows that $u_{\lambda} \in N_{\lambda}$ and, in particular, $J\left(u_{\lambda}\right) \geq \alpha_{\lambda}$. 
Next, we will show, up to a subsequence, that $u_{n} \rightarrow u_{\lambda}$ strongly in $W_{0}^{1, p}(\Omega)$ and $J\left(u_{\lambda}\right)=\alpha_{\lambda}$. From the fact $u_{n}, u_{\lambda} \in N_{\lambda},(4)$ and Fatou's Lemma, it follows that

$$
\begin{aligned}
\alpha_{\lambda} \leq & J\left(u_{\lambda}\right) \\
= & \frac{1}{p}\left\|u_{\lambda}\right\|_{\mu}^{p}-\frac{1}{p^{*}(a)} \int_{\Omega} \frac{\left|u_{\lambda}\right|^{*^{*}(a)}}{|x|^{a}}-\frac{1}{p^{*}(b)} \int_{\Omega} \frac{\left|u_{\lambda}\right|^{*}(b)}{\left|x-x_{0}\right|^{b}}-\frac{\lambda}{q} \int_{\Omega} \frac{\left|u_{\lambda}\right|^{q}}{|x|^{s}} \\
= & \frac{1}{p}\left(\int_{\Omega} \frac{\left|u_{\lambda}\right|^{p^{*}(a)}}{|x|^{a}}+\int_{\Omega} \frac{\left|u_{\lambda}\right|^{p^{*}(b)}}{\left|x-x_{0}\right|^{b}}+\int_{\Omega} \frac{\left|u_{\lambda}\right|^{q}}{|x|^{s}}\right)-\frac{1}{p^{*}(a)} \int_{\Omega} \frac{\left|u_{\lambda}\right|^{p^{*}(a)}}{|x|^{a}} \\
& -\frac{1}{p^{*}(b)} \int_{\Omega} \frac{\left|u_{\lambda}\right|^{p^{*}(b)}}{\left|x-x_{0}\right|^{b}}-\frac{\lambda}{q} \int_{\Omega} \frac{\left|u_{\lambda}\right|^{q}}{|x|^{s}} \\
= & \left.\frac{1}{p}-\frac{1}{p^{*}(a)}\right) \int_{\Omega} \frac{\left|u_{\lambda}\right|^{p^{*}(a)}}{|x|^{a}}+\left(\frac{1}{p}-\frac{1}{p^{*}(b)}\right) \int_{\Omega} \frac{\left|u_{\lambda}\right|^{p^{*}(b)}}{\left|x-x_{0}\right|^{b}} \\
& +\lambda\left(\frac{1}{p}-\frac{1}{q}\right) \int_{\Omega} \frac{\left|u_{\lambda}\right|^{q}}{|x|^{s}} \\
\leq & \lim _{n \rightarrow \infty}\left\{\left(\frac{1}{p}-\frac{1}{p^{*}(a)}\right) \int_{\Omega} \frac{\left|u_{n}\right|^{p^{*}(a)}}{|x|^{a}}+\left(\frac{1}{p}-\frac{1}{p^{*}(b)}\right) \int_{\Omega} \frac{\left|u_{n}\right|^{p^{*}(b)}}{\left|x-x_{0}\right|^{b}}\right. \\
& \left.+\lambda\left(\frac{1}{p}-\frac{1}{q}\right) \int_{\Omega} \frac{\left|u_{n}\right|^{q}}{|x|^{s}}\right\} \\
= & \lim _{n \rightarrow \infty} J\left(u_{n}\right) \\
= & \alpha_{\lambda},
\end{aligned}
$$

which implies that $J\left(u_{\lambda}\right)=\alpha_{\lambda}$ and $\lim _{n \rightarrow \infty}\left\|u_{n}\right\|_{\mu}^{p}=\left\|u_{\lambda}\right\|_{\mu}^{p}$. Standard argument shows that $u_{n} \rightarrow u_{\lambda}$ strongly in $W_{0}^{1, p}(\Omega)$. Moreover, $u_{\lambda} \in N_{\lambda}^{+}$. Otherwise, if $u_{\lambda} \in N_{\lambda}^{-}$, from Lemma 2.4 there exist unique $\tau_{\lambda}^{+}$and $\tau_{\lambda}^{-}$such that $\tau_{\lambda}^{+} u_{\lambda} \in N_{\lambda}^{+}, \tau_{\lambda}^{-} u_{\lambda} \in N_{\lambda}^{-}$and $\tau_{\lambda}^{+}<\tau_{\lambda}^{-}=1$. Since

$$
\frac{d}{d \tau} J\left(\tau_{\lambda}^{+} u_{\lambda}\right)=0 \quad \text { and } \quad \frac{d^{2}}{d \tau^{2}} J\left(\tau_{\lambda}^{+} u_{\lambda}\right)>0
$$

there exists $\bar{\tau} \in\left(\tau_{\lambda}^{+}, \tau_{\lambda}^{-}\right)$such that $J\left(\tau_{\lambda}^{+} u_{\lambda}\right)<J\left(\tau_{\lambda}^{-} u_{\lambda}\right)$. By Lemma 2.4 , we get that

$$
J\left(\tau_{\lambda}^{+} u_{\lambda}\right)<J\left(\bar{\tau} u_{\lambda}\right) \leq J\left(\tau_{\lambda}^{-} u_{\lambda}\right)=J\left(u_{\lambda}\right)
$$

which is a contradiction. Since $J\left(u_{\lambda}\right)=J\left(\left|u_{\lambda}\right|\right)$ and $\left|u_{\lambda}\right| \in N_{\lambda}^{+}$, by Lemma 2.2, we may assume that $u_{\lambda}$ is a nontrivial nonnegative solution of (1). From the strong maximum principle [19], it follows that $u_{\lambda}>0$ in $\Omega$. Finally, by (9), Hölder and Sobolev-Hardy inequalities, we obtain

$$
\begin{aligned}
0 & <\left\langle\phi_{\lambda}^{\prime}\left(u_{\lambda}\right), u_{\lambda}\right\rangle \\
& =\left(p-p^{*}(b)\right)\left\|u_{\lambda}\right\|_{\mu}^{p}-\left(p^{*}(a)-p^{*}(b)\right) \int_{\Omega} \frac{\left|u_{\lambda}\right|^{p^{*}(a)}}{|x|^{a}}-\lambda\left(q-p^{*}(b)\right) \int_{\Omega} \frac{\left|u_{\lambda}\right|^{q}}{|x|^{s}} \\
& \leq\left(p-p^{*}(b)\right)\left\|u_{\lambda}\right\|_{\mu}^{p}-\lambda\left(q-p^{*}(b)\right) \int_{\Omega} \frac{\left|u_{\lambda}\right|^{q}}{|x|^{s}} .
\end{aligned}
$$


Thus

$$
\left\|u_{\lambda}\right\|_{\mu}^{p-q}<\lambda \frac{p^{*}(b)-q}{p^{*}(b)-p} \int_{\Omega} \frac{\left|u_{\lambda}\right|^{q}}{|x|^{s}}<\lambda \frac{p^{*}(b)-q}{p^{*}(b)-p}\left(\frac{N \omega_{N} R_{0}^{N-s}}{N-s}\right)^{\frac{p^{*}(s)-q}{p^{*}(s)}} A_{\mu, s}^{-\frac{q}{p}}
$$

which implies that $\left\|u_{\lambda}\right\|_{\mu} \rightarrow 0$ as $\lambda \rightarrow 0^{+}$.

Next we will establish the existence of the second positive solution of (1) by proving that $J(u)$ satisfies the $(\mathrm{PS})_{\alpha_{\lambda}}$ condition.

Lemma 3.3 Let $\left\{u_{n}\right\}$ be a bounded sequence in $W_{0}^{1, p}(\Omega)$. If $\left\{u_{n}\right\}$ is a (PS) sequence for $J(u)$ with $c \in\left(0, \Lambda_{1}\right)$ where $\Lambda_{1}$ is defined in the introduction. Then there exists a subsequence of $\left\{u_{n}\right\}$ converging weakly to a nonzero solution solution of (1).

Proof The proof is similar to that of Corollary 4.3 in [15], and the details are omitted.

Lemma 3.4 ([14]) Assume $1<p<N, 0 \leq a<p$ and $0 \leq \mu<\bar{\mu}$. Then the problem

$$
\begin{cases}-\Delta_{p} u-\mu \frac{|u|^{p-2} u}{|x|^{p}}=\frac{|u|^{p^{*}(a)-2} u}{|x|^{a}} & \text { in } R^{N} \backslash\{0\} \\ u>0 & \text { in } R^{N} \backslash\{0\} \\ u \in D^{1, p}\left(R^{N}\right) & \end{cases}
$$

has radially symmetric ground states

$$
\bar{V}_{\varepsilon}(x)=\varepsilon^{-\frac{N-p}{p}} U_{p, \mu}\left(\frac{x}{\varepsilon}\right)=\varepsilon^{-\frac{N-p}{p}} U_{p, \mu}\left(\frac{|x|}{\varepsilon}\right), \quad \forall \varepsilon>0,
$$

satisfying

$$
\int_{R^{N}}\left(\left|\nabla \bar{V}_{\varepsilon}(x)\right|^{p}-\mu \frac{\left|\bar{V}_{\varepsilon}(x)\right|^{p}}{|x|^{p}}\right)=\int_{R^{N}} \frac{\left|\bar{V}_{\varepsilon}(x)\right|^{p^{*}(a)}}{|x|^{a}}=\left(A_{\mu, a}\right)^{\frac{N-a}{p-a}},
$$

where $U_{p, \mu}(x)=U_{p, \mu}(|x|)$ is the unique radial solution for problem (16) satisfying

$$
U_{p, \mu}(1)=\left(\frac{(N-a)(\bar{\mu}-\mu)}{N-p}\right)^{\frac{1}{p^{*}(a)-p}}
$$

and $D^{1, p}\left(R^{N}\right)=\left\{u \in L^{p^{*}}\left(R^{N}\right): \nabla u \in L^{p}\left(R^{N}\right)\right\}$. Moreover, $U_{p, \mu}(x)$ also has the following properties:

$$
\begin{aligned}
& \lim _{r \rightarrow 0} r^{a(\mu)} U_{p, \mu}(r)=c_{1}>0, \\
& \lim _{r \rightarrow+\infty} r^{b(\mu)} U_{p, \mu}(r)=c_{2}>0, \\
& \lim _{r \rightarrow 0} r^{a(\mu)+1} U_{p, \mu}^{\prime}(r)=c_{1} a(\mu) \geq 0, \\
& \lim _{r \rightarrow+\infty} r^{b(\mu)+1} U_{p, \mu}^{\prime}(r)=c_{2} b(\mu)>0,
\end{aligned}
$$


where $c_{1}$ and $c_{2}$ are positive constants depending on $p$ and $N, a(\mu)$ and $b(\mu)$ are the zeros of the function

$$
f(\tau)=(p-1) \tau^{p}-(N-p) \tau^{p-1}+\mu, \quad \tau \geq 0,0 \leq \mu<\bar{\mu},
$$

satisfying

$$
0 \leq a(\mu)<\frac{N-p}{p}<b(\mu) \leq \frac{N-p}{p-1} .
$$

Remark 2 By direct calculation, we deduce that $\tau_{\min }=\frac{N-p}{p}$ is the only minimum point of $f(\tau)$. Furthermore, $f^{\prime}(\tau)<0$ for $0<\tau<\tau_{\min }$ and $f^{\prime}(\tau)>0$ for $\tau>\tau_{\min }$. Thus, we infer that

$$
\begin{aligned}
\tau_{\min }<\frac{N}{p} \leq b(\mu) & \Longleftrightarrow f\left(\frac{N}{p}\right) \leq f(b(\mu))=0 \\
& \Longleftrightarrow 0<\mu \leq \frac{N^{p-1}\left(N-p^{2}\right)}{p^{p}} .
\end{aligned}
$$

Furthermore, by (17) we know that $b(\mu)>\frac{N}{p}$ implies $N>p^{2}$.

Lemma 3.5 ([7]) Suppose $1<p<N, 0 \leq b<p$. Then the following holds:

(i) $A_{0, b}$ is independent of $\Omega$;

(ii) $A_{0, b}$ is attained when $\Omega=R^{N}$ by the functions

$$
y_{\varepsilon}(x)=\left(\varepsilon(N-b)\left(\frac{N-p}{p-1}\right)^{p-1}\right)^{\frac{N-p}{p(p-b)}}\left(\varepsilon+\left|x-x_{0}\right|^{\frac{p-b}{p-1}}\right)^{\frac{p-N}{p-b}}
$$

for some $\varepsilon>0$. Moreover, the functions $y_{\varepsilon}(x)$ solve the equation

$$
-\Delta_{p} u=\frac{|u|^{p^{*}(b)-2} u}{\left|x-x_{0}\right|^{b}} \text { in } R^{N} \backslash\left\{x_{0}\right\}
$$

and satisfy

$$
\int_{R^{N}}\left|\nabla y_{\varepsilon}\right|^{p}=\int_{R^{N}} \frac{\left|y_{\varepsilon}\right|^{p^{*}(b)}}{\left|x-x_{0}\right|^{b}}=\left(A_{0, b}\right)^{\frac{N-b}{p-b}}
$$

Lemma 3.6 If $0 \leq \mu<\bar{\mu}, 0 \leq a, b<p$ and $1 \leq q<p$, then for any $\lambda>0$, there exists $v_{\lambda} \in$ $W_{0}^{1, p}(\Omega)$ such that

$$
\sup _{\tau \geq 0} J\left(\tau v_{\lambda}\right)<\Lambda_{1}
$$

In particular, $\alpha_{\lambda}^{-}<\Lambda_{1}$ for all $\lambda \in\left(0, \Lambda_{0}\right)$, where $\lambda_{1}$ is defined in the introduction.

Proof Now we distinguish two cases, that is, $\frac{p-a}{p(N-a)} A_{\mu, a}^{\frac{N-a}{p-a}} \leq \frac{p-b}{p(N-b)} A_{0, b}^{\frac{N-b}{p-b}}$ and $\frac{p-a}{p(N-a)} A_{\mu, a}^{\frac{N-a}{p-a}}>$ $\frac{p-b}{p(N-b)} A_{0, b}^{\frac{N-b}{p-b}}$.

Case 1. $\frac{p-a}{p(N-a)} A_{\mu, a}^{\frac{N-a}{p-a}} \leq \frac{p-b}{p(N-b)} A_{0, b}^{\frac{N-b}{p-b}}$. 
Assume $\rho>0$ is small enough such that $B(0, \rho) \subset \Omega, \varphi(x) \in C_{0}^{\infty}(\Omega), 0 \leq \varphi(x) \leq 1$, $\varphi(x)=1$ for $|x| \leq \frac{\rho}{2}, \varphi(x)=0$ for $|x| \geq \rho$. Let

$$
u_{\varepsilon}(x)=\varphi(x) \bar{V}_{\varepsilon}(x), \quad \varepsilon>0 .
$$

The following estimates are from [10] and [14]:

$$
\begin{aligned}
& \left\|u_{\varepsilon}\right\|_{\mu}^{p}=\left(A_{\mu, a}\right)^{\frac{N-a}{p-a}}+O\left(\varepsilon^{b(\mu) p+p-N}\right), \\
& \int_{\Omega} \frac{\left|u_{\varepsilon}\right|^{p^{*}(a)}}{|x|^{a}}=\left(A_{\mu, a}\right)^{\frac{N-a}{p-a}}+O\left(\varepsilon^{b(\mu) p^{*}(a)+a-N}\right), \\
& \int_{\Omega} \frac{\left|u_{\varepsilon}\right|^{q}}{|x|^{s}} \geq \begin{cases}c \varepsilon^{N-s+\left(1-\frac{N}{p}\right) q}, & q>\frac{N-s}{b(\mu)}, \\
c \varepsilon^{N-s+\left(1-\frac{N}{p}\right) q}|\ln \varepsilon|, & q=\frac{N-s}{b(\mu)}, \\
c \varepsilon^{q\left(b(\mu)+1-\frac{N}{p}\right)}, & q<\frac{N-s}{b(\mu)} .\end{cases}
\end{aligned}
$$

Now we consider the following functions:

$$
\begin{aligned}
g(\tau) & =J\left(\tau u_{\varepsilon}\right) \\
& =\frac{\tau^{p}}{p}\left\|u_{\varepsilon}\right\|_{\mu}^{p}-\frac{\tau^{p^{*}(a)}}{p^{*}(a)} \int_{\Omega} \frac{\left|u_{\varepsilon}\right|^{p^{*}(a)}}{|x|^{a}}-\frac{\tau^{p^{*}(b)}}{p^{*}(b)} \int_{\Omega} \frac{\left|u_{\varepsilon}\right|^{p^{*}(b)}}{\left|x-x_{0}\right|^{b}}-\lambda \frac{\tau^{q}}{q} \int_{\Omega} \frac{\left|u_{\varepsilon}\right|^{q}}{|x|^{s}},
\end{aligned}
$$

and

$$
\bar{g}(\tau)=\frac{\tau^{p}}{p}\left\|u_{\varepsilon}\right\|_{\mu}^{p}-\frac{\tau^{p^{*}(a)}}{p^{*}(a)} \int_{\Omega} \frac{\left|u_{\varepsilon}\right|^{p^{*}(a)}}{|x|^{a}} .
$$

Using the definitions of $g$ and $u_{\varepsilon}$, we get

$$
g(\tau)=J\left(\tau u_{\varepsilon}\right) \leq \frac{\tau^{p}}{p}\left\|u_{\varepsilon}\right\|_{\mu}^{p}, \quad \text { for all } \tau \geq 0 \text { and } \lambda>0 .
$$

Combining this with (19) and letting $\varepsilon \in(0,1)$, there exists $\tau_{0} \in(0,1)$ independent of $\varepsilon$ such that

$$
\sup _{0 \leq \tau \leq \tau_{0}} g(\tau)<\frac{p-a}{p(N-a)} A_{\mu, a}^{\frac{N-a}{p-a}}, \quad \text { for all } \lambda>0 \text { and all } \varepsilon \in(0,1) .
$$

On the other hand, by the fact that

$$
\max _{\tau \geq 0}\left(\frac{\tau^{p}}{p} B_{1}-\frac{\tau^{p^{*}(a)}}{p^{*}(a)} B_{2}\right)=\frac{p-a}{p(N-a)} B_{1}^{\frac{N-a}{p-a}} B_{2}{ }^{-\frac{N-p}{p-a}}, \quad B_{1}>0, B_{2}>0,
$$

and from (19) and (20), we obtain that

$$
\begin{aligned}
\max _{\tau \geq 0} \bar{g}(\tau) & =\frac{p-a}{p(N-a)}\left\|\nabla u_{\varepsilon}\right\|_{\mu}^{\frac{p(N-a)}{p-a}}\left(\int_{\Omega} \frac{\left|u_{\varepsilon}\right|^{p^{*}(a)}}{|x|^{a}}\right)^{-\frac{N-p}{p-a}} \\
& =\frac{p-a}{p(N-a)}\left(\left(A_{\mu, a}\right)^{\frac{N-a}{p-a}}+O\left(\varepsilon^{b(\mu) p+p-N}\right)\right)^{\frac{N-a}{p-a}}
\end{aligned}
$$




$$
\begin{aligned}
& \times\left(\left(A_{\mu, a}\right)^{\frac{N-a}{p-a}}+O\left(\varepsilon^{b(\mu) p^{*}(a)+a-N}\right)\right)^{-\frac{N-p}{p-a}} \\
= & \frac{p-a}{p(N-a)} A_{\mu, a}^{\frac{N-a}{p-a}}+O\left(\varepsilon^{b(\mu) p+p-N}\right) .
\end{aligned}
$$

Hence as $\lambda>0,1 \leq q<p$, by (24) we have that

$$
\begin{aligned}
\sup _{\tau \geq \tau_{0}} g(\tau) & \leq \sup _{\tau \geq \tau_{0}}\left(\bar{g}(\tau)-\lambda \frac{\tau^{q}}{q} \int_{\Omega} \frac{\left|u_{\varepsilon}\right|^{q}}{|x|^{s}}\right) \\
& \leq \frac{p-a}{p(N-a)} A_{\mu, a}^{\frac{N-a}{p-a}}+O\left(\varepsilon^{b(\mu) p+p-N}\right)-\lambda \frac{\tau_{0}^{q}}{q} \int_{\Omega} \frac{\left|u_{\varepsilon}\right|^{q}}{|x|^{s}} .
\end{aligned}
$$

(i) If $1 \leq q<\frac{N-s}{b(\mu)}$, then by (21), we obtain that

$$
\int_{\Omega} \frac{\left|u_{\varepsilon}\right|^{q}}{|x|^{s}} \geq C \varepsilon^{q\left(b(\mu)+1-\frac{N}{p}\right)}
$$

and since $b(\mu)>\frac{N-p}{p}, q<p$, we obtain

$$
b(\mu) p+p-N>q\left(b(\mu)+1-\frac{N}{p}\right) .
$$

Combining this with (22) and (25), for any $\lambda>0$, we can choose $\varepsilon_{\lambda}$ small enough such that

$$
\sup _{\tau \geq 0} g(\tau)=\sup _{\tau \geq 0} J\left(\tau u_{\varepsilon_{\lambda}}\right)<\frac{p-a}{p(N-a)} A_{\mu, a}^{\frac{N-a}{p-a}} .
$$

(ii) If $\frac{N-s}{b(\mu)} \leq q<p$, then by (21) and $b(\mu)>\frac{N-p}{p}$, we obtain

$$
\int_{\Omega} \frac{\left|u_{\varepsilon}\right|^{q}}{|x|^{s}} \geq \begin{cases}c \varepsilon^{N-s+\left(1-\frac{N}{p}\right) q}, & q>\frac{N-s}{b(\mu)}, \\ c \varepsilon^{N-s+\left(1-\frac{N}{p}\right) q}|\ln \varepsilon|, & q=\frac{N-s}{b(\mu)},\end{cases}
$$

and $b(\mu) p+p-N>N-s+\left(1-\frac{N}{p}\right) q$. Combining this with (22) and (25), for any $\lambda>0$, we can choose $\varepsilon_{\lambda}$ small enough such that

$$
\sup _{\tau \geq 0} J\left(\tau u_{\varepsilon_{\lambda}}\right)<\frac{p-a}{p(N-a)} A_{\mu, a}^{\frac{N-a}{p-a}} .
$$

From (26) and (27), we obtain the result in Case 1 by taking $v_{\lambda}=u_{\varepsilon_{\lambda}}$.

Case 2. $\frac{p-a}{p(N-a)} A_{\mu, a}^{\frac{N-a}{p-a}}>\frac{p-b}{p(N-b)} A_{0, b}^{\frac{N-b}{p-b}}$.

Let

$$
\begin{aligned}
& C_{\varepsilon}=\left(\varepsilon(N-b)\left(\frac{N-p}{p-1}\right)^{p-1}\right)^{\frac{N-p}{p(p-b)}}, \\
& U_{\varepsilon}(x)=\frac{y_{\varepsilon}(x)}{C_{\varepsilon}} .
\end{aligned}
$$


Consider $\varphi(x) \in C_{0}^{\infty}(\Omega), 0 \leq \varphi(x) \leq 1, \varphi(x)=1$ for $\left|x-x_{0}\right| \leq \frac{R}{2}, \varphi(x)=0$ for $\left|x-x_{0}\right| \geq R$, where $B\left(x_{0}, R\right) \subset \Omega$. Denote

$$
\begin{aligned}
& v_{\varepsilon}(x)=\varphi(x) U_{\varepsilon}(x), \quad \text { for all } \varepsilon>0, \\
& w_{\varepsilon}(x)=\frac{v_{\varepsilon}(x)}{\left(\int_{\Omega} \frac{\left|v_{\varepsilon}\right|^{*}(b)}{\left|x-x_{0}\right|^{b}}\right)^{\frac{1}{p^{*}(b)}}},
\end{aligned}
$$

such that

$$
\int_{\Omega} \frac{\left|w_{\varepsilon}\right|^{p^{*}(b)}}{\left|x-x_{0}\right|^{b}}=1 .
$$

Then we can obtain the following results by the methods used in [7]:

$$
\begin{aligned}
& \int_{\Omega}\left|\nabla w_{\varepsilon}\right|^{p}=A_{0, b}+O\left(\varepsilon^{\frac{N-p}{p-b}}\right), \\
& \int_{\Omega}\left|w_{\varepsilon}\right|^{q} \geq \begin{cases}c \varepsilon^{\frac{q(N-p)}{p(p-b)}}, & q<\frac{N(p-1)}{N-p} \\
c \varepsilon^{\frac{q(N-p)}{p(p-b)}}|\ln \varepsilon|, & q=\frac{N(p-1)}{N-p}, \\
c \varepsilon^{\frac{(p-1)(p N-q(N-p))}{p(p-b)}}, & q>\frac{N(p-1)}{N-p} .\end{cases}
\end{aligned}
$$

Observing that $w_{\varepsilon}$ concentrates on $x=x_{0}$ when $\varepsilon>0$ is small enough, we can easily estimate

$$
\int_{\Omega} \frac{\left|w_{\varepsilon}\right|^{q}}{|x|^{s}} \geq \begin{cases}c \varepsilon^{\frac{q(N-p)}{p(p-b)}}, & q<\frac{N(p-1)}{N-p} \\ c \varepsilon^{\frac{q(N-p)}{p(p-b)}}|\ln \varepsilon|, & q=\frac{N(p-1)}{N-p} \\ c \varepsilon^{\frac{(p-1)(p N-q(N-p))}{p(p-b)}}, & q>\frac{N(p-1)}{N-p} .\end{cases}
$$

Especially, when $q=p$, we have

$$
\int_{\Omega} \frac{\left|w_{\varepsilon}\right|^{p}}{|x|^{p}} \geq \begin{cases}c \varepsilon^{\frac{N-p}{p-b}}, & p^{2}>N \\ c \varepsilon^{\frac{N-p}{p-b}}|\ln \varepsilon|, & p^{2}=N \\ c \varepsilon^{\frac{p(p-1)}{p-b}}, & p^{2}<N .\end{cases}
$$

Now we consider the following function:

$$
\begin{aligned}
h(\tau)= & J\left(\tau w_{\varepsilon}\right) \\
= & \frac{\tau^{p}}{p} \int_{\Omega}\left(\left|\nabla w_{\varepsilon}\right|^{p}-\mu \frac{\left|w_{\varepsilon}\right|^{p}}{|x|^{p}}\right)-\frac{\tau^{p^{*}(a)}}{p^{*}(a)} \int_{\Omega} \frac{\left|w_{\varepsilon}\right|^{p^{*}(a)}}{|x|^{a}} \\
& -\frac{\tau^{p^{*}(b)}}{p^{*}(b)} \int_{\Omega} \frac{\left|w_{\varepsilon}\right|^{p^{*}(b)}}{\left|x-x_{0}\right|^{b}}-\lambda \frac{\tau^{q}}{q} \int_{\Omega} \frac{\left|w_{\varepsilon}\right|^{q}}{|x|^{s}}
\end{aligned}
$$

Since $\lim _{\tau \rightarrow+\infty} h(\tau)=-\infty$ and $\lim _{\tau \rightarrow 0^{+}} h(\tau)<0$, combining this with Remark 1, we get that $\sup _{\tau \geq 0} h(\tau)$ is attained for some $0<\tau_{0}<+\infty$. Together with (23) and (28)-(31), we 
calculate that

$$
\begin{aligned}
& h(\tau) \leq h\left(\tau_{0}\right) \\
& \leq \frac{\tau_{0}^{p}}{p} \int_{\Omega}\left(\left|\nabla w_{\varepsilon}\right|^{p}-\mu \frac{\left|w_{\varepsilon}\right|^{p}}{|x|^{p}}\right)-\frac{\tau_{0}^{p^{*}(b)}}{p^{*}(b)}-\lambda \frac{\tau_{0}^{q}}{q} \int_{\Omega} \frac{\left|w_{\varepsilon}\right|^{q}}{|x|^{s}} \\
& \leq \frac{p-b}{p(N-b)}\left(A_{0, b}+O\left(\varepsilon^{\frac{N-p}{p-b}}\right)\right)^{\frac{N-b}{p-b}}-\frac{\tau_{0}^{p}}{p} \int_{\Omega} \mu \frac{\left|w_{\varepsilon}\right|^{p}}{|x|^{p}}-\lambda \frac{\tau_{0}^{q}}{q} \int_{\Omega} \frac{\left|w_{\varepsilon}\right|^{q}}{|x|^{s}} \\
& \leq \frac{p-b}{p(N-b)} A_{0, b}^{\frac{N-b}{p-b}}+O\left(\varepsilon^{\frac{N-p}{p-b}}\right)- \begin{cases}c \varepsilon^{\frac{N-p}{p-b}}, & p^{2}>N, \\
c \varepsilon^{\frac{N-p}{p-b}}|\ln \varepsilon|, & p^{2}=N, \\
c \varepsilon^{\frac{p(p-1)}{p-b}}, & p^{2}<N,\end{cases} \\
& - \begin{cases}c \varepsilon^{\frac{q(N-p)}{p(p-b)}}, & q<\frac{N(p-1)}{N-p}, \\
c \varepsilon^{\frac{q(N-p)}{p(p-b)}}|\ln \varepsilon|, & q=\frac{N(p-1)}{N-p}, \\
c \varepsilon^{\frac{(p-1)(p N-q(N-p))}{p(p-b)}}, & q>\frac{N(p-1)}{N-p} .\end{cases}
\end{aligned}
$$

(i) If $p^{2} \geq N$, then we have that $\frac{N-p}{p-b}>\frac{q(N-b)}{p(p-b)}$. By (32), for any $\lambda>0$, we can choose $\varepsilon_{\lambda}$ small enough such that

$$
h\left(\tau_{0}\right)=\sup _{\tau \geq 0} J\left(\tau w_{\varepsilon_{\lambda}}\right)<\frac{p-b}{p(N-b)} A_{0, b}^{\frac{N-b}{p-b}}-c \varepsilon^{\frac{q(N-p)}{p(p-b)}}<\frac{p-b}{p(N-b)} A_{0, b}^{\frac{N-b}{p-b}} .
$$

(ii) If $p^{2}<N$, then we have that $\frac{N-p}{p-b}>\frac{p(p-1)}{p-b}$. By (32), for any $\lambda>0$, we can choose $\varepsilon_{\lambda}$ small enough such that

$$
h\left(\tau_{0}\right)=\sup _{\tau \geq 0} J\left(\tau w_{\varepsilon_{\lambda}}\right)<\frac{p-b}{p(N-b)} A_{0, b}^{\frac{N-b}{p-b}}-c \varepsilon^{\frac{p(p-1)}{p-b}}<\frac{p-b}{p(N-b)} A_{0, b}^{\frac{N-b}{p-b}} .
$$

From (i) and (ii), we obtain the result in Case 2 by taking $v_{\lambda}=w_{\varepsilon_{\lambda}}$.

From Lemma 2.4, the definition of $\alpha_{\lambda}^{-}$and (18), for any $\lambda \in\left(0, \Lambda_{0}\right)$, we obtain that there exists $\tau_{\lambda}^{-}>0$ such that $\tau_{\lambda}^{-} v_{\lambda} \in N_{\lambda}^{-}$and

$$
\alpha_{\lambda}^{-} \leq J\left(\tau_{\lambda}^{-} v_{\lambda}\right) \leq \sup _{\tau \geq 0} J\left(\tau v_{\lambda}\right)<\Lambda_{1}
$$

The proof is thus complete.

Now we establish the existence of a local minimum of $J(u)$ on $N_{\lambda}^{-}$.

Theorem 3.7 Assume that $N \geq 3,0 \leq \mu<\bar{\mu}, 0 \leq a, b<p$ and $1 \leq q<p$. If $\lambda \in\left(0, \frac{q}{p} \Lambda_{0}\right)$, then there exists $U_{\lambda} \in N_{\lambda}^{-}$such that

(i) $J\left(U_{\lambda}\right)=\alpha_{\lambda}^{-}$;

(ii) $U_{\lambda}$ is a positive solution of (1).

Proof If $\lambda \in\left(0, \frac{q}{p} \Lambda_{0}\right)$, then by Lemmas 2.5(ii), 3.1(ii), and 3.6, there exists a (PS) $\alpha_{\lambda}^{-}$sequence $\left\{u_{n}\right\} \subset N_{\lambda}^{-}$in $W_{0}^{1, p}(\Omega)$ for $J(u)$ with $\alpha_{\lambda}^{-} \in\left(0, \Lambda_{1}\right)$. Since $J(u)$ is coercive on $N_{\lambda}$, we get that 
$\left\{u_{n}\right\}$ is bounded in $W_{0}^{1, p}(\Omega)$. From Lemma 3.3, there exists a subsequence still denoted by $\left\{u_{n}\right\}$ and a nontrivial solution $U_{\lambda} \in W_{0}^{1, p}(\Omega)$ of (1) such that $u_{n} \rightarrow U_{\lambda}$ weakly in $W_{0}^{1, p}(\Omega)$. First we prove that $U_{\lambda} \in N_{\lambda}^{-}$. Arguing by contradiction, we assume $U_{\lambda} \in N_{\lambda}^{+}$. Since $N_{\lambda}^{-}$is closed in $W_{0}^{1, p}(\Omega)$, we have $\left\|u_{\lambda}\right\|_{\mu}<\liminf _{n \rightarrow \infty}\left\|u_{n}\right\|_{\mu}$. Thus by Lemma 2.4 , there exists a unique $\tau_{\lambda}^{-}$such that $\tau_{\lambda}^{-} U_{\lambda} \in N_{\lambda}^{-}$. From Remark $1, u_{n} \in N_{\lambda}^{-},\left\|U_{\lambda}\right\|_{\mu}<\liminf _{n \rightarrow \infty}\left\|u_{n}\right\|_{\mu}$, and (4), we can deduce that

$$
\alpha_{\lambda}^{-} \leq J\left(\tau^{-} U_{\lambda}\right)<\lim _{n \rightarrow \infty} J\left(\tau_{\lambda}^{-} u_{n}\right) \leq \lim _{n \rightarrow \infty} J\left(u_{n}\right)=\alpha_{\lambda}^{-} .
$$

This is a contradiction. Thus $U_{\lambda} \in N_{\lambda}^{-}$.

Next, by the same argument as that in Theorem 3.2, we get that $u_{n} \rightarrow U_{\lambda}$ strongly in $W_{0}^{1, p}(\Omega)$ and $J\left(U_{\lambda}\right)=\alpha_{\lambda}^{-}>0$ for all $\lambda \in\left(0, \frac{q}{p} \Lambda_{0}\right)$. Since $J\left(U_{\lambda}\right)=J\left(\left|U_{\lambda}\right|\right)$ and $\left|U_{\lambda}\right| \in N_{\lambda}^{-}$, by Lemma 2.2 we may assume that $U_{\lambda}$ is a nontrivial nonnegative solution of (1). Finally, by the maximum principle, we obtain that $U_{\lambda}$ is a positive solution of (1).

The proof of Theorem 1.1 Now we complete the proof of Theorem 1.1. Part (i) of Theorem 1.1 immediately follows from Theorem 3.2. When $0<\lambda<\frac{q}{p} \Lambda_{0}<\Lambda_{0}$, by Theorems 3.2 and 3.7, we obtain that (1) has at least two positive solutions $u_{\lambda}$ and $U_{\lambda}$ such that $u_{\lambda} \in N_{\lambda}^{+}$, $U_{\lambda} \in N_{\lambda}^{-}$. Since $N_{\lambda}^{+} \cap N_{\lambda}^{-}=\emptyset$, this implies that $u_{\lambda}$ and $U_{\lambda}$ are distinct. This completes the proof of Theorem 1.1.

\section{Acknowledgements}

The authors would like to thank the referees for their valuable comments and suggestions which improved the original manuscript.

\section{Funding}

The project was supported by National Natural Science Foundation of China (Grant No. 11871212) and North China University of Water Resources and Electric power (Grant No. 70495).

Availability of data and materials

Not applicable.

\section{Competing interests}

The authors declare that they have no competing interests.

\section{Authors' contributions}

All the authors contributed to each part of this study equally and approved the final version of the manuscript.

\section{Publisher's Note}

Springer Nature remains neutral with regard to jurisdictional claims in published maps and institutional affiliations.

Received: 16 April 2019 Accepted: 1 August 2019 Published online: 13 August 2019

\section{References}

1. Brezis, H., Nirenberg, L.: Positive solutions of nonlinear elliptic equations involving critical Sobolev exponent. Commun. Pure Appl. Math. 36, 437-477 (1983)

2. Cao, D., Han, P.: Solutions for semilinear elliptic equations with critical exponents and Hardy potential. J. Differ. Equ. 205, 521-537 (2004)

3. Cao, D., Peng, S.: A note on the sign-changing solutions to elliptic problems with critical Sobolev and Hardy terms. J. Differ. Equ. 193, 424-434 (2003)

4. Ekeland, I.: On the variational principle. J. Math. Anal. Appl. 47, 324-353 (1974)

5. Gao, W., Peng, S.: An elliptic equation with combined critical Sobolev-Hardy terms. Nonlinear Anal. TMA 65 1595-1612 (2006)

6. Gazzola, F., Ruf, B.: Lower-order perturbations of critical growth nonlinearities in semilinear elliptic equations. Adv. Differ. Equ. 2, 555-572 (1997)

7. Ghoussoub, N., Yuan, C.: Multiple solutions for quasi-linear PDEs involving the critical Sobolev and Hardy exponents. Trans. Am. Math. Soc. 352, 5703-5743 (2000)

8. Guo, Q., Niu, P., Cui, X.: Existence of sign-changing solutions for semilinear singular elliptic equations with critical Sobolev exponents. J. Math. Anal. Appl. 361, 543-557 (2010) 
9. Guo, Q., Niu, P., Dou, J.: Multiplicity of solutions for singular semilinear elliptic equations with critical Hardy-Sobolev exponents. Appl. Anal. Discrete Math. 2, 158-174 (2008)

10. Han, P.: Quasilinear elliptic problems with critical exponents and Hardy terms. Nonlinear Anal. TMA 61, 735-758 (2005)

11. Hsu, T.: Multiplicity results for $p$-Laplace with critical nonlinearity of concave-convex type and sign-changing weight functions. Abstr. Appl. Anal. 2009, Article ID 652109 (2009)

12. Hsu, T:: Multiple positive solutions for a quasilinear elliptic involving critical Sobolev-Hardy exponents and concave-convex nonlinearities. Nonlinear Anal. TMA 74, 3934-3944 (2011)

13. Jannelli, E.: The role played by space dimension in elliptic critical problems. J. Differ. Equ. 156, 407-426 (1999)

14. Kang, D: On the quasilinear elliptic problems with critical Sobolev-Hardy exponents and Hardy terms. Nonlinear Anal. TMA 68, 1973-1985 (2008)

15. Li, Y., Guo, Q., Niu, P.: Global compactness results for quasilinear elliptic problems with combined critical Sobolev-Hardy terms. Nonlinear Anal. TMA 74, 1445-1464 (2011)

16. Li, Y., Guo, Q., Niu, P.: The existence of solutions for quasilinear elliptic problems with combined critical Sobolev-Hardy terms. J. Math. Anal. Appl. 388, 525-538 (2012)

17. Liang, S., Zhang, J.: Multiplicity of solutions for a class of quasilinear elliptic equation involving the critical Sobolev and Hardy exponents. Nonlinear Differ. Equ. Appl. 17, 55-67 (2010)

18. Pohozaev, S.: Eigenfunctions of the equation $\Delta u+\lambda f(u)=0$. Sov. Math. Dokl. 5, 1408-1411 (1965)

19. Vazquez, J.: A strong maximum principle for some quasilinear elliptic equations. Appl. Math. Optim. 12, 191-202 (1984)

\section{Submit your manuscript to a SpringerOpen ${ }^{\circ}$ journal and benefit from:}

- Convenient online submission

- Rigorous peer review

Open access: articles freely available online

- High visibility within the field

Retaining the copyright to your article

Submit your next manuscript at $\boldsymbol{~ s p r i n g e r o p e n . c o m ~}$ 